\title{
Measurements of Double Differential Cross-Sections for He at Intermediate Energy
}

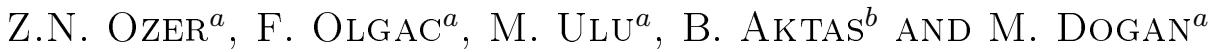 \\ ${ }^{a}$ Afyon Kocatepe University, Department of Physics, e-Col Laboratory, Afyonkarahisar, Turkey \\ ${ }^{b}$ Gebze High Technology Institute, Department of Physics, Gebze, Turkey
}

\begin{abstract}
Detailed studies on ionization of atoms and molecules by electron impact have made numerous contributions to understanding of the structure of atoms and molecules including the interaction between particles. After a ionizing collision a scattered electron, an ejected electron and a recoil ion is formed. Doubly differential cross-sections give detailed information for one of the outgoing electrons. In this study, we report the angular distribution of double differential cross-sections of ejected electrons from He in intermediate energy region.
\end{abstract}

DOI: 10.12693/APhysPolA.125.341

PACS: $34.80 . \mathrm{Dp}$

\section{Introduction}

Generally, atomic and molecular physics leads to discovering the structure of matter in atomic or molecular level and explaining the natural laws. These goals are precisely succeed by collision methods. Applications of the results from collision physics are mainly important in atmospheric science, laser refinement, and meteorological phenomena. An intensive effort of experimental and theoretical work has been devoted in recent years to the study of ionization differential cross-sections of atoms by electron impact. The ionization of rare gas atoms, in particular the cross-sections obtained by ground state ionization, are considered benchmark data [1-5]. Doubly differential cross-sections (DDCS) of ionization, $\mathrm{d}^{2} \sigma\left(E_{\mathrm{b}}, \theta_{\mathrm{b}}\right)$, as a function of ejected energy, $E_{\mathrm{b}}$ and the angle of the ionized electron, $\theta_{\mathrm{b}}$, carry valuable information on the both collision dynamics and the internal structure of atomic systems.

The DDCS is proportional to the number of incident probe particle scattered with an energy range and momentum variation into a solid angle $\Delta \Omega$ and represented by $\mathrm{d}^{2} \sigma /\left(\mathrm{d} \Omega_{\mathrm{b}} \mathrm{d} E_{\mathrm{b}}\right)$.

DDCS, differential in energy and direction of one of the outgoing electrons, can be obtained through the measurement of the energy and angular distributions of outgoing electrons with only one detector.

Opal et al. [1], Shyn and Sharp [2], Müller-Fiedler et al. $[3,4]$ carried out the measurements of DDCS of e-He ionization. Experimental DDCS of helium shows differences in the shape of angular and energy distributions. Röder et al. [6] presented experimental and theoretical DDCSs of He for a wide range of impact energies. The measured energy range for these studies were at intermediate energies varying as $100-2000 \mathrm{eV}$, however no experimental data exists in literature for the kinematics of this study.

A significant advance in the theory of electron impact ionization was done by Brauner et al. [7]. They presented a model (Brauner, Briggs and Klar - BBK) for description of final state three-body Coulomb wave function fully compatible with asymptotic boundary condition. Chen and $\mathrm{Xu}[8]$ presented DDCS calculations of $\mathrm{He}$ at low energies in comparison to convergent close-coupling (CCC) method. Biswas and Sinha [9] calculated triple differential cross-sections (TDCS) and DDCSs of He for asymmetric geometry at intermediate and medium energies in comparison with corresponding experiments.

In this paper, DDCS in which only one existing electron is detected, for electron impact ionization of helium have been measured in a crossed-beam experiment. Angular dependences of cross-sections for ejected electrons are presented. Ejected electron energy and angle dependent differential cross-sections for ionization of helium by electron collision have been evaluated at fixed incident electron energies of $55,80,150$, and $250 \mathrm{eV}$.

\section{Experimental setup}

The apparatus used to measure the DDCSs is described in Refs. $[10,11]$. The electron beam that is produced by electron gun is placed in vacuum chamber and it is guided to reaction region by electrostatic fields. The background pressure inside chamber is $8.8 \times 10^{-8} \mathrm{mbar}$ and it increases to $7.8 \times 10^{-6} \mathrm{mbar}$ while the experiment is operating. The electron beam was produced by an electron gun that during the present measurements has been operated at $55,80,150$, and $250 \mathrm{eV}$ energies and at currents $\approx 3 \mu \mathrm{A}$. The diameter of the electron beam at the scattering center was around $2 \mathrm{~mm}$. The electron beam current was continuously monitored by a Faraday cup placed on the axis of the electron gun at $50 \mathrm{~mm}$ from the collision center. The effusive gaseous beam was let into the chamber via a needle orthogonal to the electron beam.

The DDCS can be measured using a cross beam type experiment. The electron beam crosses by the target gas at the interaction region in the perpendicular plane. The outgoing electrons are detected by electron energy analyzer after the collision. Electrons of the correct selected energy are detected and amplified using channeltron electron multipliers (CEM). 


\section{Results and discussion}

It is essential for DDCS measurements to obtain accurate knowledge on the energies of incident and scattered electrons. On accounts of this calibration of energy and angle it is important for experimental setup not to be irresponsive.
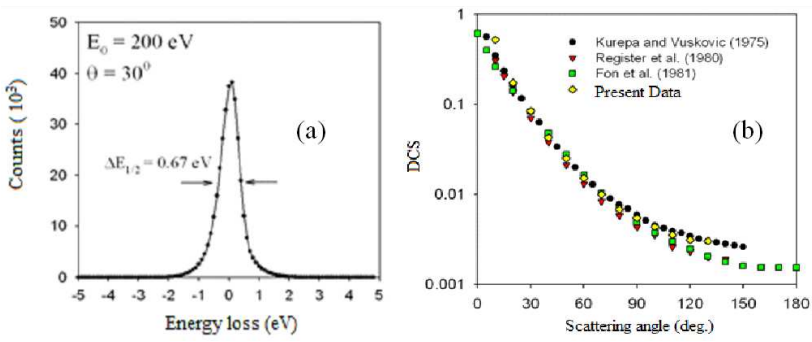

Fig. 1. (a) Elastic peak of $\mathrm{He}$ at $200 \mathrm{eV}$, (b) elastic DCS of He at $200 \mathrm{eV}$ in comparison with the data of Kurepa and Vuskovic (1975), Register et al. (1980), Fon et al. (1981).

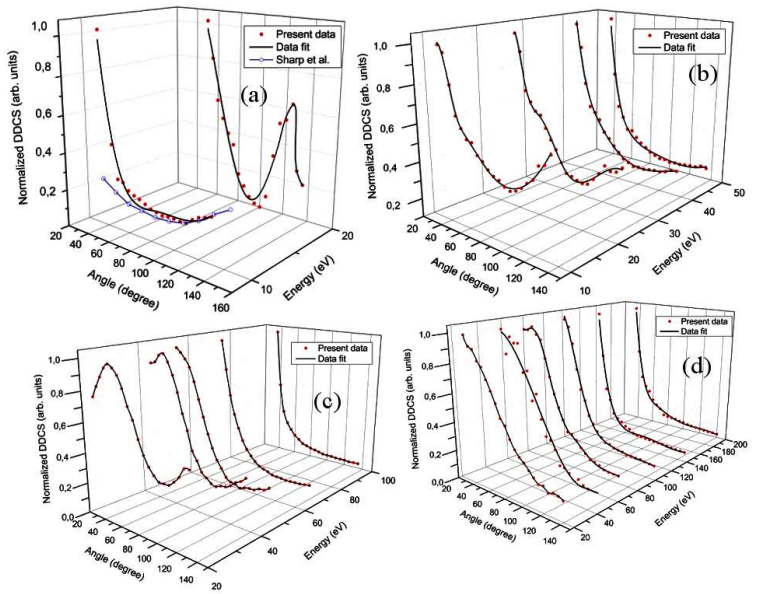

Fig. 2. DDCS spectra for 55 (a), 80 (b), 150 (c), and 250 (d) eV electron impact ionization of He. The DDCS results of He for $250 \mathrm{eV}$ impact energy at 20,40, 50, 70, $110,150 \mathrm{eV}$, for $150 \mathrm{eV}$ impact energy at 20, 40, 50, 70, and $100 \mathrm{eV}$ ejected electron energies, for $80 \mathrm{eV}$ electron impact energy at 10,20,40, and $50 \mathrm{eV}$ and at $55 \mathrm{eV}$ electron impact energy for 10 and $20 \mathrm{eV}$ ejected electron energy. The present experimental data is shown within filled symbols and the experimental data of Sharp et al. is shown within non-filled circles.

The calibrations are done by measuring energy loss spectra of He. In Fig. 1, an example of energy loss spectrum of $\mathrm{He}$, is presented for $20 \mathrm{eV}$ kinetic energy. This technique is used to mark the exact location of the peaks for the level of interest and is also important to determine the resolution of the spectrometer energy. In addition to elastic peak, elastic differential cross-section (DCS) measurements are given in comparison with the data of Kurepa and Vuskovic [12], Register et al. [13], Fon et al.
[14] at $200 \mathrm{eV}$ incident electron energy. The results are in agreement with the data in the literature.

DDCS of outgoing electrons from $\mathrm{He}$ by 55, 80, 150, and $250 \mathrm{eV}$ electron impact have been measured by a crossed beam method. The ejection energies varied in the range 10-150 eV. Measured electron energy increased as the impact energy increased, since more data is available for higher impact energies.

The angular range of ejected electrons measured in the range of $40-130^{\circ}$. Figure 2 shows the DDCS results of He. The DDCS results are found to be in good agreement with the experimental data of Sharp et al. [2] for $55 \mathrm{eV}$ impact and $10 \mathrm{eV}$ ejected energy.

The outgoing electrons are mostly scattered isotropically in all directions. Ejected electrons with higher energies produce some structure in the cross-section due to a binary collision between the incident electron and an electron from the target of He. It is possible to say that DDCS as a function of the ejected (or scattered) angle may provide valuable information on the mechanisms of ionization for different regions of collision kinematics.

\section{Acknowledgments}

This work was supported by the Scientific and Technological Research Council of Turkey (TÜBİTAK) through grant 109T738.

\section{References}

[1] C.B. Opal, E.C. Beaty, W.K. Peterson, At. Data 4, 209 (1972).

[2] T.W. Shyn, W.E. Sharp, Phys. Rev. A 19, 557 (1979).

[3] R. Müller-Fiedler, K. Jung, H. Ehrhardt, J. Phys. B 19, 1211 (1986).

[4] R. Müller-Fiedler, P. Schlemmer, K. Jung, H. Ehrhardt, Z. Phys. A 320, 89 (1985).

[5] T. Rösel, P. Schlemmer, J. Röder, L. Frost, K. Jung, H. Ehrhardt, Z. Phys. D 23, 359 (1992).

[6] J. Röder, H. Ehrhardt, I. Bray, D.V. Fursa, J. Phys. B 30, 1309 (1997).

[7] M. Brauner, J.S. Briggs, H. Klar, J. Phys. B 22, 2265 (1989).

[8] Z. Chen, K. Xu, J. Phys. B 32, 1259 (1999).

[9] R. Biswas, C. Sinha, Phys. Rev. A 51, 3766 (1995).

[10] Z.N. Ozer, H. Chaluvadi, M. Ulu, M. Dogan, B. Aktas, D. Madison, Phys. Rev. A 87, 042704 (2013).

[11] M. Dogan, M. Ulu, O. Sise, J. Electron. Spectrosc. Relat. Phenom. 161, 58 (2007).

[12] M.V. Kurepa, L. Vuskovic, J. Phys. B 8, 2067 (1975).

[13] D.F. Register, S. Trajmar, S.K. Srivastava, Phys. Rev. A 21, 1134 (1980).

[14] W.C. Fon, K.A. Berrington, A. Hibbert, J. Phys. B 14, 307 (1981). 